\title{
Zur Entstehung der Aspiration des Thorax bei der Geburt.
}

Von

\section{J. $\mathbf{B} \boldsymbol{e} \mathbf{n} \mathbf{t} \in \mathbf{i}$}

in Halle a./S.

Nachdem von mir nachgewiesen war ${ }^{1}$ ), dass die Aspiration des Thorax bei der Geburt durch eine bleibende Erhebung der Rippen in Folge der ersten Athemzüge herbeigeführt wird, wurden von mir zwei Möglichkeiten zur Erklärung dieses Vorganges hingestellt. Die eine bestand in der Annahme, dass durch die erste kräftige Inspiration eine Ueberdehnung der exspiratorisch wirkenden elastischen Apparate, der Muskeln, Bänder u. s. w., stattfinde, so dass die Gleichgewichtslage des Thorax sich zu Gunsten der Inspiration verschiebe, die andere ging dahin, dass die Rippen in ihren Gelenken vermöge eines Mechanismus, welcher nach Art der Sperrzähne oder in ähnlicher Weise wirkt, in einer höheren Gleichgewichtslage festgehalten wïrden. Es wird sich aus den weiter unten angeführten Aaseinandersetzungen ergeben, dass von diesen beiden Annahmen die erstere höchst wahrscheinlich die richtige ist.

Bevor ich dazu übergehe, ist es nothwendig, eine andere Theorie zurückzuweisen, welehe unterdess von L. Hermann und Kelle $\mathrm{r}^{2}$ ) der meinigen entgegengestellt worden ist. Ich habe es bis jetzt unterlassen, auf diesen Gegenstand zurüickzukommen, da das zur Untersuchnng nöthige Material mir immer nur in längeren Zwischenpausen zu Gebote stand und ich auch durch anderweitige Beschäftigung davon abgelenkt war. Ich bin aber jetzt doch genöthigt, darauf einzugehen, da ich sehe, dass J. Rosenthal sich in dem Hermann'schen Handbuche ${ }^{3}$ ) der Physiologie auf die Seite von Hermann gestellt hat, und da dieses Handbuch doch wohl den Anspruch erhebt, die allgemeinguiltigen Anschanungen in der Physiologie für einige Zeit zu fixiren, so wird es mir ge-

1) Dieses Archiv, Bd. XVII, S. 617.

2) Dieses Archiv, Bd. XX, S. 365.

3) Bd. IV, 2, S. 228 и. 229.

E. Pflüger, Archiv f. Physiologie. Bd. zxvmI, 
stattet sein, dem Leser den Stand der Sache und die Ergebnisse meiner weiteren Beobachtungen etwas ausführlich darzulegen.

Hermann hat in der bezeichneten Arbeit nicht etwa neue Beobachtungen über das Verhalten des Thorax beim Foetus oder Todtgeborenen angestellt, um dadurch die von mir gemachten Annahmen iber die Entstehung der Aspiration direkt zu widerlegen, sondern er hat sich zunächst darauf beschränkt, zu erklären, dass ihm meine Theorien „einen etwas gezwungenen Eindruck" machten und hat dieselben durch eine andere Theorie ersetzt. Dieselbe besteht erstens in der Annahme, dass der Thorax des Foetus vermöge seiner Entwickelung im Uterus an sich das Bestreben habe, sich elastisch auszudehnen, und zweitens darin, dass diese elastische Ausdehnung nur durch die Adhäsion und Verklebung der Bronchialwände aneinander verhindert werde. Sobald aber dieser Widerstand durch die erste Inspiration iberwunden sei, bleibe der Thorax in erweiterter Lage stehen.

Es kann nun keinem Zweifel unterliegen, dass in der atelektatischen Lunge eine Adhäsion der Bronchialflächen stattfinde, und dass diese dem Lufteintritt einen gewissen Widerstand biete; es wäre auch kaum nöthig gewesen, durch die Versuche Keller's an atelektatisch gemachten Kaninchenlungen das Vorhandensein einer solchen Adhäsion zu beweisen. Aber welchen Beweis hat Hermann dafür erbracht, dass in Folge seines Wachsthnms der Thorax des Foetus das Bestreben habe, sich vermöge elastischer Kräfte auszudehnen?

Es lässt sich durch eine kurze Ueberlegung zeigen, dass ein solches Verhalten des Thorax beim Foetus höchst unwahrscheinlich ist: Setzen wir also den Fall, dass der Thorax sich auszudehnen strebe, so würde allerdings die Möglichkeit vorliegen, dass die Adhäsion der Bronchialwände dem Eintritt von Luft einen Widerstand biete, sobald das Kind geboren ist. Aber Hermann hat ganz ausser Acht gelassen, dass der Foetus vor der Geburt sich nicht in der Luft, sondern unter Wasser befindet.

Wenn wir zwei feuchte Membranen aneinanderlegen, so haften sie in der Luft aneinander und bieten der Lösung einen gewissen Widerstand. Wenn wir aber die Membranen in Wasser eintauchen, so lösen sie sich bekanntlich ganz von selbst. Die Bronchialflächen der foetalen Lunge verhalten sich aber während der ganzen Zeit ihrer Entwickelung ganz ebenso wie zwei anein- 
ander liegende in Wasser befindliche Membranen. Wenn Hermann und Keller daher versucht hätten, ihre atelektatischen Lungen mit Wasser anstatt mit Luft aufzublasen, so würden sie ohne Zweifel gefunden haben, dass hierzu nar ein Minimum von Druck erforderlich gewesen wäre. Also würde die Lunge des Foetus, welche durch Mund und Nase mit dem Fruchtwasser communicirt, sich mit letzterem anfïllen müssen, sobald eine Ansaugung von Seiten des Thorax stattfände. - Nach der Theorie Hermann's miisste jeder Foetus in seinem eigenen Fruchtwasser ertrinken.

Um allen Einwänden gegen diese Folgerungen der Hermann'schen Theorie zu begegnen, will ich zum Ueberfluss die Sache physikalisch zergliedern. Es handelt sich in diesem Falle um die Adhäsion zweier Oberflächen aneinander, welche mit Flüssigkeit benetzt sind, nicht etwa um die Adhäsion von trockenen Oberflächen fester Körper; denn es befindet sich zwischen den Oberflächen der Bronchialmembranen mindestens eine capillare Schicht von Flüssigkeit. Ausserdem befinden sich die Membranen noch im Zustande der Imbibition. Es ist daher in diesem Falle von der Anziehung der festen Oberflächen gegeneinander gänzlich abzusehen. Das Aneinanderhaften wird vielmehr einzig und allein dureh die Cohäsion der benetzenden Flüssigkeit hervorgerufen; denn bei der Trennung der Oberflächen voneinander werden nur die Flüssigkeitsmoleküle voneinander gerissen, während dieselben durch Adhäsion an den Oberflächen haften bleiben ${ }^{1}$ ).

Nun ist es klar, dass wenn die adhärirenden Körper sich in der Flüssigkeit selbst befinden, zwischen die getrennten Flüssigkeitsmoleküle sofort neue Molekïle eintreten, also nur eine Verschiebung der Moleküle gegeneinander eintritt. Hierza ist aber nur eine verbältnissmässig kleine Kraft erforderlich, welche von der innern Reibung der Flïssigkeit abhängt.

Um das Gesagte durch einen einfachen Versuch zu erläutern, nehme man zwei Glasplatten, etwa zwei längliche Objectgläser, und lege sie gut befeuchtet kreuzweis übereinander, ohne sie fest aufeinander zu pressen. In der Luft wird die untere Platte an der oberen fest haften und dem Abreissen merklichen Widerstand darbieten. Tancht man sie aber in Wasser ein, indem man die

1) S. Versuche von Gay-Lussac über Adhäsion der Flüssigkeiten an der Oberfläche fester Körper. Wüllner's Lehrbuch der Physik, Bd. I, S.261. 
obere Platte an der schmäleren überstehenden Seite mit den Fingern hält, so fällt die untere entweder von selbst oder bei einem ganz schwachen Anstoss ab. Hat man dagegen die Glasplatten fest aufeinander gepresst, so bleiben sie auch unter Wasser haften und setzen dem Abreissen deutlichen Widerstand entgegen, weil nun ausserdem noch die Adhäsion der festen Oberflächen in Wirkung getreten ist.

Man bedecke ferner die beiden Glasplatten mit zwei imbibirten Membranen aus Thierblase und lege sie in derselben Weise mit ihren Membranen aufeinander. Nun kann man sie ziemlich stark gegeneinander pressen, trotzdem fallen sie unter Wasser durch einen leichten Anstoss ab. Hier kommt also, trotz starker Pressung, die Adbäsion 'fester Oberflächen nur wenig zur Wirkung, wahrscheinlich desshalb, weil an der Oberfläche der imbibirten Membranen eine dickere Flïssigkeitsschicht gebunden ist, als an der Oberfläche fester Körper. Wir werden also hieraus schliessen dürfen, dass, wenn die Bronchialflächen der foetalen Lunge sogar aneinandergepresst wären, - eine ganz unnatürliche Voraussetzung, zu der gar kein Grund vorliegt - sie dem Eindringen des Fruchtwassers doch nur wenig Widerstand darbieten würden.

Nun könnte man freilich als einen verstärkenden Factor für die Adhäsion der Bronchialwände die Zähigkeit der secernirten Zwischenflüssigkeit in Anspruch nehmen. In der That würden ja zwei Membranen, welche miteinander durch eine klebrige Flüssigkeit verbunden sind, auch unter Wasser stärker aneinanderhaften. Aber auch diese Kraft wird keine sehr beträchtliche werden können, wie z. B. bei zwei mit Gummi arabicum bestrichenen Membranen, welche sich unter Wasser mit Leichtigkeit lösen. Dass aber die Bronchialflüssigkeit beim Foetus sehr viel zäher sein sollte als das Fruchtwasser, ist nicht sehr wahrscheinlich, da bei der allgemeinen Unthätigkeit aller. Drüsen eine merkliche Schleimabsonderung auf den Bronchialflächen kaum anzunehmen ist. Ferner müsste bei der lang andauernden Communication der Bronchialfliussigkeit mit dem Fruchtwasser durch Diffusion eine allmähliche Ablösung der Wände unvermeidlich sein, wenn man nicht der Sehnelligkeit der Diffusion entsprechend, einen Nachschub von zähem Sekrete annehmen wollte. Endlich muss man bedenken, dass eine starke Verklebung der Bronchialwände mit zähem Sekrete, welche der Thorax-Aspiration Widerstand leisten sollte, 
nach der Geburt dem Beginn der Laftathmung einen sehr erheblichen Widerstand entgegensetzen würde, - eine im höchsten Grade unzweckmässige Einrichtung, welche wir nach dem Darwin'schen Princip der Natur nicht zutrauen könnten.

Nun könnte man ferner die Hermann'sche Theorie von der elastischen Spannung des foetalen Thorax noch durch die Annahme zu halten suchen, dass der Eintritt von Fruchtwasser in den ansaugenden Thorax durch eine ventilartige Vorrichtung, z. B. durch den Verschluss des Kehlkopfs mit Hülfe des Kehldeckels verhittet werde. Ein solcher Verschluss würde denkbar sein, wenn der Kehldeckel beim Foetus constant die Lage innehielte, welche er beim Schlingen annimmt. Ueber die Lage des Kehldeckels beim Foetus ist mir bisher Nichts bekannt geworden und babe ich anch selbst noch keine Gelegenheit gehabt, darïber Beobachtungen anzustellen. Aber bekannt ist es, dass der Foetus Schlingbewegungen macht und mit dem Fruchtwasser Haare und Epidermisfetzen in den Darm befördert. Es geht daraus hervor, dass die Zungen-, Gaumen- und Rachenmuskulatur keineswegs in Ruhe bleibt und es ist daher wenig wahrscheinlich, dass der Kehldeckel in einer unbeweglichen Lage verharrt. Wehe dem Foetus, welcher unvorsichtiger Weise seinen Kehldeckel ein wenig heben würde! - Die Gefahr des Ertrinkens wäre eine sehr drohende.

Ich möchte mich indessen nicht darauf beschränken, durch die vorangeschickte Diskussion der Frage die Hermann'sche Theorie als sehr unwahrscheinlich, ja eigentlich unmöglich, zurtickgewiesen zu haben, sondern befinde mich in der günstigen Lage, das Experimentum crucis angeben zu können, welches über die vorliegende Frage entscheidet.

Es ist klar, dass, wenn der Thorax des Foetus sich vermöge seiner elastischen Kräfte auszudehnen strebte, die Frweiterung desselben sofort eintreten müsste, sobald wir ihn von Aussen eröffnen würden. Haben wir adso an einem Todtgeborenen die Pleurahöhlen von der Brustwand aus geöffnet, so müsste nun Luft in dieselben eindringen, während der Thorax sich erweitern miisste. Wer aber jemals die Sektion eines Todtgebornen angestellt hat, der wird wissen, dass hierbei von irgend welchen Anzeichen einer Thoraxerweiterung Nichts eintritt. Die Thoraxwandungen bleiben vielmehr in ihrer vorigen Lage, es bildet sich kein Cavam zwischen Lunge und Thoraxwand, vielmehr füllen Lunge und Herz den 
Thoraxraum vollständig aus und von einem Eindringen der Luft ist Nichts zu merken. Ein elastischer Zug, weleher die Rippen heben sollte, kommt also unter diesen Verhältnissen gar nicht zur Wirkung, während doch jeder Widerstand von Seiten der Lunge in diesem Falle aufgehoben ist.

Nun könnte man auch hier noch einwenden, dass es ebenfalls die Adhäsion der beiden Pleuraflächen sei, welche dem Abheben des Thorax Widerstand leiste. Aber dieser Einwand lässt sich leicht widerlegen; denn haben wir an dem Todtgeborenen durch Aufblasung der Lunge die Aspiration des Thorax hergestellt und öffnen nun die Pleurahöhlen, so löst sich ja die Lunge vermöge ihrer Elasticität mit Leichtigkeit von der Thoraxwand ab. Die Kraft aber, mit welcher das geschieht, muisste genau gleich sein derjenigen Kraft, mit welcher sich die Thoraxwand von der atelektatischen Lunge abheben würde, wenn überhaupt eine solche Kraft vorhanden wäre.

Um den Einwand wegen der Adhäsion der Pleuraflächen noch experimentell zu widerlegen, könnte man die Eröffnung des Thorax unter Wasser vornehmen. Indess babe ich es vorgezogen, den Versuch in folgender Weise anzustellen: Es wird ein kleines Manometerrohr mit einer Stichcanüle auf einer Seite verbunden und mit Lacmuslösung so weit gefüllt, dass die Canüle ganz damit angefüllt ist während die Flüssigkeit in freien Schenkeln eine entsprechende Höhe einnimmt. Dann wird der freie Schenkel miteinem Kautschuckröhrchen versehen und dieses zugeklemmt. Stechen wir die Caniile durch die Thoraxwand in die Pleurahöhle ein, so wird nach dem Abnehmen der Klemme die Flässigkeit eingesogen werden, wenn der Thorax aspirirt. Dies geschah auch sehr deutlich bei einem getödteten Kaninchen, an welchem die Canüle des Manometers rechterseits zwischen 4. und 5. Rippe eingestochen wurde. Im freien Schenkel sank die Flüssigheit erst sehnell um $25 \mathrm{~mm}$., dann langsam um $19 \mathrm{~mm}$., also im Ganzen um $44 \mathrm{~mm}$. Die Stichcanüle wurde erst schräg unter die Haut eingesenkt, dann mit einem Faden um Haut und Gewebe umschnürt und dann schräg durch einen Intercostalraum in die Brtsthöhle eingestossen, so dass kein Lufteintritt stattfinden konnte.

An einer männlichen Kindesleiche, welche nach vorgenommener Perforation ohne Lebenszeichen geboren war, fand die Beobachtung in der beschriebenen Weise statt. Die Caniule wurde 
rechterseits zwischen 5. und 6. Rippe eingestochen. Nachdem das Manometer geöffnet war, blieb die Flüssigkeit an der Skala ruhig stehen. Nun wurden von der Trachea aus Einblasungen in die Lunge vorgenommen. Hierbei verlegt sich die Canüle leicht und schlüpft auch wohl wieder aus der Brusthöhle heraus. Nachdem dieselbe wieder reponirt war, sank die Flüssigkeit im freien Schenkel des Manometers allmählich um $50 \mathrm{~mm}$.

Nachdem die Canüle mit Manometer links in derselben Höhe eingestochen war, fand auch hier noch ein Sinken der Flüssigkeit um $22 \mathrm{~mm}$. statt.

Nun wurde noch ein anderes Manometer mit der Trachea verbunden und nach Eröffnung beider Pleurahöhlen ein Druck von $46 \mathrm{~mm}$. daran beobachtet.

Bei der Sektion zeigte sich, dass die Stichöffnung rechts in der Pleurahöhle sich befand and die Lungenoberfläche stellenweise durch Lacmus gefärbt war. Links war der Stich in den Herzbeutel gerathen.

In einem zweiten Falle war der Foetus intrauterin durch Nabelschnurverfall abgestorben. Ein mit der Trachea verbundenes Manometer mit Lacmuslösung zeigte bei Compressionen des Thorax kleine Schwankungen von $1-2 \mathrm{~mm}$. an, ein Zeichen, dass die Lunge nicht mehr ganz atelektatisch war und dass intrauterine Athembewegungen, wenn auch nur sehr schwache, stattgefunden, welche den Thorax und Lunge nur wenig erweitert hatten. Das Ausbleiben solcher Schwankungen bei Thoraxcompression ist ein sicheres Zeichen für vollkommene Atelektase der Lungen. In diesem Falle war also etwas Fruchtwasser in die Luftwege eingedrungen, was sich auch durch die weitere Beobachtung bestätigte.

Es wird nun das kleine Manometer durch die Stichcanüle mit der rechten Pleurahöhle zwischen 3. und 4. Rippe in beschriener Weise in Verbindung gesetzt. Beim Oeffnen des Manometers blieb auch hier der Stand der Wassersäule derselbe. Um zu ermitteln, ob nicht etwa die Canïle verstopft sei, wurde das Manometer so gehoben, dass im freien Schenkel ein Ueberdruck entstand und in Folge dessen die Fluissigkeit um $4,5 \mathrm{~mm}$. sank. Dann blieb dieselbe ruhig stehen. Eine Ansaugung fand also keineswegs statt. Nun wurden wieder von der Trachea aus Lufteinblasungen vorgenommen, während das Manometer gesehlossen war. Nachdem die Canüle wieder reponirt und das Manometer geöffnet war, sank die Flüssigkeit allmählich um $30 \mathrm{~mm}$. 
Bei den Einblasungen entstanden exspiratorische Rasselgeräusche und es entleerte sich eine mit Meconium vermischte Flüssigkeit aus der Trachea. Der Foetus hatte also in Folge der Nabelschnurcompression schwache Athembewegungen gemacht, welche aber nicht im Stande gewesen waren, Thorax und Lunge in erheblichem. Grade auszudehnen und eine merkliche Aspiration des Thorax noch nicht hergestellt hatten. Eine dritte Beobachtung dieser Art wurde leider durch Verstopfung der Einsticheanüle vereitelt.

Ich glaube nach diesen Versuchen mit Bestimmtheit den Satz aussprechen zu können, dass sich am Thorax des Foetus eine elastische Spannung, welche denselben ausaudehnen strebe, nicht nachweisen lässt.

Nachdem nan somit der Hermann'schen Theorie jede Basis entzogen ist, haben wir noch einen hierher gehörigen Punkt aufzuklären. Am Schlusse seiner Arbeit sagt Hermann: „Die von Bernstein anfgeworfene Frage ist somit in der einfachsten Weise beantwortet. Da der Widerstand der atelektatischen Lunge gegen den Lufteintritt relativ gross ist, so genuigen die elastischen Kräfte, mit welchen der Thorax in seine natürliche Exspirationsstellung uiberzugehen strebt, nachdem der intrauterine Druck durch die Geburt beseitigt ist, nicht zur Entfaltung von Lunge und Thorax, sondern es müssen grössere Kräfte einwirken, wie sie durch die Inspirationsmuskeln oder durch künstliche Aufblasung entwickelt werden." Es scheint hiernach so, als ob dem intrauterinen Druck die Rolle augeschrieben wird, durch Compression der Entfaltung des Thorax entgegenzuwirken.

Sollte dies gemeint sein, so wäre wiederum gänzlich übersehen, dass der Foetus sich in einer geschlossenen mit Flüssigkeit gefitllten Höhle befindet. Der Druck, unter welchem die Fliussigkeit darin steht, kann beliebig hoch sein, er würde niemals verhindern können, dass der Thorax sich vermöge elastischer Kräfte erweitere und Fruchtwasser in die Lunge einsange. Der Druck der Fliussigkeit auf die Innenfläche der Lunge ist gerade so gross wie auf die Aussenfläche des Thorax, und da eben so viel Fliissigkeit in die Lunge ein- als aus der Eihöhle abströmen würde, das Volumen des Ganzen also dasselbe bleiben würde, so wäre der herrschende Druck für den Vorgang ganz gleichgültig.

Nun könnte man freilich, wie schon oben besprochen, auch für diesen Fall noch die Voraussetzung hinzufïgen, dass ein Ven- 
tilverschluss an den Athemwegen vorhanden wäre, welcher durch den intrauterinen Druck gesperrt würde. Ein solcher Verschluss etwa mit Hülfe des Kehldeckels ist aber, wie schon oben bemerkt, nicht anzunehmen. Indessen könnte man noch daran denken, dass der intranterine Druck durch Compression der Trachea einen ventilartigen Schluss hervorbringe. Was nun das Verhalten der Trachea anbetrifft, so habe ich beobachtet, dass dieselbe beim Foetus resp. Todtgeborenen, wie nicht anders zu erwarten, mit ihren Wänden von vorn nach hinten platt aneinander gelegt ist, während sie nach der Lufteinblasung sofort klaffend bleibt. Es kann aber nicht angenommen werden, dass ihre knorplichen Wände so schlaff seien, dass sie durch den intrauterinen Druck noch dazu unter der Hautbedeckung comprimirt wuirden. Denn wenn dies der Fall wäre, so wïrden ja intrauterine Athembewegungen nicht im Stande sein, Fruchtwasser in die Lungen hineinzusaugen, wie dies in Wirklichkeit geschieht. Das hypothetische Kehldeckelventil könnte ja allenfalls durch Muskelaktion gehoben werden, die Trachea aber müsste bei der Inspiration vermöge des vergrösserten Druckunterschiedes innerhalb und ausserhalb des Thoraxraumes um so stärker comprimirt werden.

Das Verhalten der Trachea ist iibrigens an sich von Interesse und verdient besondere Beachtung. Dieselbe wird, wie oben erwähnt, keineswegs durch einen äussern Druck beim Foetus platt zusammengedrüekt, sondern erbält sich auch beim Todtgeborenen, nachdem sie blossgelegt und durchschnitten ist, in dieser Form, bis sie durch Lufteinblasung klaffend gemacht wird. Es ist also klar, dass ihre Wände sich in zwei verschiedenen Gleichgewichtslagen befinden. In der foetalen Gleichgewichtslage sind die Knorpelhalbringe gestreckt, nachdem Luft- (oder auch Fruchtwasser) eingetreten ist, sind sie ringförmig nach, vorn convex gebogen. Die Beobachtung an einer solchen Trachea hat mir gezeigt, dass die Knorpel so biegsam sind, dass sie in jeder Gestalt, in welche man sie bringt, verharren. Die aufgeblasene und wieder zusammengedriickte Trachea bleibt wieder platt- und bandförmig, wie sie vorher gewesen und behält diese Form von selbst, nicht etwa vermöge der Adhäsion ihrer Schleimhautflächen, da auch die abgeschnittenen Knorpel sich durch Biegung in jede Form bringen lassen. Es ist also klar, dass die foetale Trachea so lange ihre bandförmige Gestalt behält, bis sie durch einen auf ihre Innenfläche ausgeübten Druck aufgeblasen wird. Ich denke mir daher, 
dass mit den ersten Athemzïgen zunächst durch das spaltförmige mit Flüssigkeit gefüllte Lumen der Trachea ein mässiges Quanium Luft in die Lunge eindringt, und dass dann dureh den Exspirationsdruck besonders beim Schreien die Trachea so weit aufgeblasen wird, wie wir sie beim Neugeborenen vorfinden. Erst dann wird eine ansgiebige Füllung der Lungen mit Luft eintreten können, und vielleicht liegt in diesem Vorgange zum Theil die Bedeutung des ersten Schreiens, mit welchem der Neugeborene die Welt begrüsst. Endlich kann man noch daran denken, dass durch Contraktion der glatten Ringmuskulatur, welche vielleicht mit den ersten Athembewegungen in Thätigkeit gerieth, das Aufklaffen der Tra- chea unterstïtzt wird.

Wie sich diese Vorgänge nun auch in Wirklichkeit gestalten mögen, soviel steht fest, dass die Trachea nicht einen ventilartigen Abschluss beim Foetus hergeben kann. Ueberhaupt wird ja eine solche Annahme gänzlich hinfällig, nachdem wir nachgewiesen haben, dass der Thorax des Foetus in der Ruhe gar keine Aspiration ausübt.

Hermann hebt ferner mir gegenüber den Unterschied der zusammengesunkenen Lunge und der atelektatischen Lunge hervor, indem er sagt: „Es hätte vor Allem die Frage aufgestellt und beantwortet werden miissen, warum ändert die erste Entfaltung das Lungenvolumen bleibend, warum macht sie bleibenden Luftgehalt? ehe man die Mechanik des Thorax mit hineinzog."

Mir schien diese Frage mit der Entstehung der Aspiration des Thorax in gar keinem direkten Zusammenhange zu stehen und ïberhaupt höchst einfach zu sein. Wenn man eine ganz zusammengefaltete Blase, die wir etwa der atelektatischen Lunge vergleichen können, durch Aufblasen ausdehnt und dann die Luft wieder ausströmen lässt, so faltet sie sich nicht wieder von selbst zusammen, sondern bleibt in schlaffem Zustande mit etwas Luft gefüllt. Ebenso verhält sich die nach Oeffnung des Thorax sich zusammenziehende Lunge. Dass man diesen Luftrest durch Druek nicht aus ihr entfernen kann, kommt wohl daher, abgesehen von Knickungen der Bronchien, dass die kleinen Bronchien sich durch den Druck früher schliessen, bevor sich die Alveolen ganz entleert haben.

Ich möchte übrigens bei dieser Gelegenheit noch erwähnen, dass $\mathrm{Bricke}^{1}$ ) angiebt, die Lunge ziehe sich vollkommen zusammen, so dass sie in Wasser geworfen untersinke, wenn man

1) Vorlesungen über Physiologie, 3. Aufl, Bd. I, p. 430. 
den Thorax an einem lebenden Thiere eröffnet habe. Er erklärt dieses Verhalten durch die Contraktion der Muskelfasern, mit welchen die Bronchien und nach Molesch ott's und seinen eigenen Beobachtungen auch die Wände der Infundibula ausgestattet sind, während im Cadaver keine vollständige Zusammenziehung zu Stande komme. Ich habe hierüber keine eignen Versuche angestellt, doch wenn sich die Angabe bestätigte ${ }^{1}$ ), so würde dieses Verfahren atelektatische Lungen herzustellen einfacher und sicherer sein als das von Hermann angewendete. Freilich müsste man sich erst davon uiberzeugen, dass alle Luft entfernt sei.

Nachdem ich nun glaube nachgewiesen zu haben, dass der Thorax des Neugeborenen in Folge der Athembewegungen eine nene Gleichgewichtslage annimmt, die er vorher nicht besessen hat, handelt es sich darum, die Mechanik dieses Vorganges zu verstehen.

Fïr die eine der von mir angeführten Möglichkeiten, für eine sperrzahnähnliche Einrichtung oder eine im gleichen Sinne wirkende Klemmung an den Costo vertebral-Gelenken, habe ich bei der genaneren Untersuchung derselben beim Foetus keine sicheren Anlaltspunkte gefunden. Das Gelenk zwischen Tubereulum costae und Proc. transvers. zeigt beim Foetus eine fast plane oder nur wenig concave Fläche am Proc. transvers. Der Gelenkkopf des Tuberculum dagegen zeigt eine von unten nach oben hin zunehmende Krümmung. Wenn man durch dieses Gelenk senkrecht gegen Querfortsatz und Rippe einen Schnitt legt, während man die Rippe im Zusammenhang mit dem sonst unverletzten Thorax lässt, so erkennt man, dass vorzugsweise nur der untere flachere und grössere Abschnitt des Gelenkköpfchens mit der Gelenkfläche in Berthrung ist, der obere convexere dagegen von der Gelenkkapsel bedeckt wird. Wenn nun eine Drehung der Rippen nach oben stattfindet um eine Axe, welche durch die beiden Costovertebralgelenke geht, so kommen die convexeren Stellen des Gelenkköpfchen mit der Gelenkfläche in Berührung. Ich habe daher Anfangs daran gedacht, ob nicht durch dieses Verhalten eine Klemmung in dem Gelenke zu Stande käme, welche die Riickkehr der Rippen in die alte Lage verhindern könnte. Indessen war es mir nicht möglich hierfür einen Nachweis zu ftihren, da die Beweg-

1) Eine hierauf bezügliche Angabe finde ich bei Prochaska, Physiologie, 1820 (Wien), S. 286, wo bemerkt ist, dass die Lunge bei Eröffnung der Brust eines lebenden Thieres bis auf einen kleinen Umfang zusammenfalle. 
lichkeit der Rippen in diesem Gelenk nach oben wie nach unten hin anscheinend eine gleichmässig freie ist. Ich musste hiernach also die erste Möglichkeit fallen lassen.

Ich wende mich nun zur zweiten Möglichkeit'1), die ich in meiner ersten Arbeit vorangestellt hatte. Dieselbe geht auch von der Voraussetzung aus, dass der Thorax des Foetus und alle mit ihm verbundenen Theile in einem elastischen Gleichgewichtszustande wachsen. Wenn nun eine kräftige Inspiration erfolgt, so werden alle diejenigen Organe, Muskeln, Bänder u. s. w., stark gedehnt, welche in exspiratorischer Richtung ziehen. Diese Organe sind aber keine vollkommen elastischen Körper, sondern sie zeigen nach einer stärkeren Dehnung einen Dehnungsrückstand, der um so grösser ist, je weiter ihre Elastizitätsgrenze überschritten ist. Die Folge davon muss sein, dass die Gleichgewichtslage des Thorax nach der inspiratorischen Richtung hin vorrïckt, welche von nun ab beibehalten wird.

Man wird gegen diese Theorie der Ueberdehnung, wie ich sie kurz nennen will, vielleicht das Bedenken erheben, dass eine starke Exspiration auch eine Ueberdehnung der inspiratorischen Organe hervorbringen möchte, und so den alten Gleichgewichtszustand wieder herstellen könnte. Wir wissen jedoch, dass wenn die Lunge einmal mit Luft gefiillt ist, dieselbe durch Druck niemals wieder ganz aus ihr entfernt werden kann, weil sich die kleinen Bronchien früher schliessen, bevor die Alveolen sich ganz entleert haben. Es ist daher der Exspiration hierdurch eine Grenze gesetzt, bevor noch der Thorax die foetale Lage wieder erreicht hat. Aber selbst wenn er in die foetale Lage wieder zuritickehren würde, so würde auch hierdureh eine Ueberdehnung der inspiratorischen Organe nicht hervorgebracht werden, denn diese sind ja in dem Zustande der tiefsten Exspirationsstellung gewachsen. Im Uebrigen ist beim Neugeborenen zu so tiefen Exspirationen wohl keine Veranlassung vorhanden, denn diese müssten bei weiter Stimmritze vorgenommen werden. Beim Schreien aber ist die Stimmritze verengt und die Lunge bleibt bis zur nächsten Inspiration immer mit einem bedeutenden Luftrest gefuillt.

Neben der Ueberdehnung könnte man noch folgenden Umstand. mit in Betracht ziehen:

Im Uterus befindet sich die Wirbelsäule in einer sehr gekrümmten Lage, wodurch die Rippen in die tiefste Exspirations-

1) Diese ist in dem Handbuche von Rosenthal nicht erwähnt worden. 
stellung gebracht werden. Nach der Geburt streckt sich die Wirbelsäule beträchtlich und verstärkt dadureh die inspiratorisch ziehenden elastischen Kräfte. Dieselben sind aber trotzdem nicht im Stande, die Entfaltung des Thorax und der Lunge zu bewirken, sei es, dass sie zu schwach sind, um die Reibungswiderstände zu iiberwinden, sei es, dass die von Hermann hervorgehobene Adhäsion der innern Lungenflächen, die ja jedenfalls vorhanden ist und hierbei in Betracht kommen könnte, zu viel Widerstand bietet. Immerhin kann sich dieser Antheil an Kraft, der erst bei der Geburt entsteht, an der Erzengung der Thoraxaspiration betheiligen. Bei Thieren, bei welchen die Lagerungsverhältnisse des Foetus andre sind, dürfte dieser Umstand wohl wenig in's Gewicht fallen. Wenigstens habe ich an dem trächtigen Uterus einer Hündin vor Kurzem beobachtet, dass die Jungen sehr frei und beweglich darin gelagert sind, so dass durch die Geburt keine grosse Aenderungen in der Rippenstellung zu erwarten sind.

Man könnte nun schliesslich die Forderung stellen, dass für die Ueberdehnung der exspiratorischen Apparate des Thorax durch die ersten Respirationen ein exsperimenteller Beweis beigebracht werde. Indess, nachdem wir die andern Möglichkeiten einer Erklärung des Vorganges, soweit wir solche auffinden konnten, ausgeschlossen haben, bleibt, wie mir scheint, kaum etwas Anderes übrig, als die Theorie der Ueberdehnung als die im höchsten Grade wahrscheinliche zu acceptiren. Man sieht ein, dass es keine geringen Schwierigkeiten machen wïrde, an einem exspiratorisch ziehenden Organe die Ueberdehnung direkt zu beobachten. Nehmen z. B. an, wir hätten am Todtgeborenen den M. rectus abdom. präparirt und bezeichneten uns auf seiner Länge zwei Punkte, so müssten wir zunächst die Entfernung dieser Punkte voneinander in dem völlig ungedehnten, also von dem einen Insertionspunkte losgetrennten Muskel kennen. Wir müssten ferner die Entfernung beider Punkte voneinander kennen, nachdem der in seiner natiirlichen Verbindung gebliebẹne Muskel durch kräftige Lufteinblasungen in die Lunge gedehnt und dann erst von einem Insertionspunkte abgetrennt worden ist. An der Differenz beider Entfernungen vor und nach der Dehnung würde die Ueberdehnung zu erkennen sein. Beides kann man aber an demselben Muskel begreiflicherweise nicht ausführen. Man könnte sich allenfalls noch dadurch helfen, dass man die eine Messung an dem Muskel der 
einen, die andere an dem Muskel der andern Seite vornimmt. Sollte ich daher Gelegenheit zu einer solchen Beobachtung haben, so würde ich es in dieser Weise versuchen, obgleich ich mir nicht verhehle, dass sich hier viele Fehlerquellen der Messung entgegenstellen.

Es sei mir gestattet, noch eine kurze Bemerkung iiber die praktische Bedeutung der Sache anzufïgen. Die Methoden der künstlichen Respiration bei asphyktisch Geborenen werden, soweit mir bekannt, von den Geburtshelfern verschiedenartig gehandhabt. Allgemein anerkannt ist wohl die Wirksamkeit der Lufteinblasungen durch einen in die Trachea eingefibhrten Catheter. Von keiner grossen Wirkung dagegen wird meines Erachtens das blosse Comprimiren und wieder Freigeben des Thorax sein können, da Thorax und Lungen noch gar nicht in dem Maasse erweitert sind, um hierbei ergiebige Volumsschwankungen zu erleiden. Das Comprimiren kann freilich den günstigen Erfolg haben, das durch intrauterine Athembewegungen aspirirte Fruchtwasser aus Trachea und Bronchien zu entfernen und dafür etwas Luft eintreten zu lassen. Aber wenn die intranterinen Athmungen wegen Verlegung der Luftwege kein Fruchtwasser eingesogen oder aus irgend einem Grunde nicht stattgefunden hatten, so sind Bronchien und Lungen noch vollkommen atelektatisch und die Compression kann gar keine Volumsschwankung der Lunge hervorrufen, also auch beim Freigeben keinen Eintritt von Luft zur Folge haben. Es muss vielmehr der Compression nothwendigerweise eine passive Erweiterung des Thorax nachfolgen, wenn eine ausreichende Luftzufuhr stattfinden soll. Es ist aber beim Neugeborenen hier der ganz besondere Umstand zu beachten, dass die Aspiration des Thorax noch gänzlich fehlt, und dass es daher von grossem Erfolge sein mïsste, wenn wir dieselbe künstlich zu Stande bringen könnten. Da es sich nun aus meinen Untersuchungen ergeben hat, dass vornehmlich die Erhebung der Rippen die Aspiration des Thorax herstellt, so möchte ich den Geburtshelfern den Vorschlag machen, es zu versuchen, durch einen geeigneten Handgriff die Rippen in die Höhe zu schieben. Dies kann vielleicht mit Erfolg geschehen, indem man mit der Hand unter den Rand der letzten Rippenknorpel greift und nach oben hin einen Zug ausuibt. Auch andre bekannte Manipulationen werden hierzu beitragen können, wie das Erheben der Arme, oder die von B. S. Schultze angegebene Methode des Schwingens, indessen müsste eine solche, welche die Hebung der Rippen direkt erzielt, am wirksamsten sein. 